\title{
Brain growth in the NICU: critical periods of tissue-specific expansion
}

\author{
Lillian G Matthews ${ }^{1}$, Brian H Walsh ${ }^{1}$, Clare Knutsen ${ }^{2}$, Jeffrey J Neil ${ }^{3}$, Christopher D Smyser ${ }^{2}$, Cynthia E Rogers $^{2}$ and \\ Terrie $\mathrm{E}$ Inder $^{1}$
}

OBJECTIVE: To examine, using serial magnetic resonance imaging (MRI), total and tissue-specific brain growth in verypreterm (VPT) infants during the period that coincides with the early and late stages of the third trimester.

METHODS: Structural MRI scans were collected from two prospective cohorts of VPT infants ( $\leq 30$ weeks of gestation). A total of $51 \mathrm{MRI}$ scans from $18 \mathrm{VPT}$ subjects were available for volumetric analysis. Brain tissue was classified into cerebrospinal fluid, cortical gray matter, myelinated and unmyelinated white matter, deep nuclear gray matter, and cerebellum. Nine infants had sufficient serial scans to allow comparison of tissue growth during the periods corresponding to the early and late stages of the third trimester.

RESULTS: Tissue-specific differences in ex utero brain growth trajectories were observed in the period corresponding to the third trimester. Most notably, there was a marked increase in cortical gray matter expansion from 34 to 40 weeks of postmenstrual age, emphasizing this critical period of brain development.

CONCLUSION: Utilizing serial MRI to document early brain development in VPT infants, this study documents regional differences in brain growth trajectories ex utero during the period corresponding to the first and second half of the third trimester, providing novel insight into the maturational vulnerability of the rapidly expanding cortical gray matter in the NICU.

$\mathbf{T}$ he fetal brain undergoes rapid growth from the second trimester onward, with a 20 -fold increase in brain volume occurring between 20 weeks of gestation and term (3840 weeks of gestation) (1). Importantly, rates of brain growth are not consistent across this period, with cross-sectional fetal magnetic resonance imaging (MRI) studies demonstrating that the greatest period of expansion occurs during the third trimester (1). For very-preterm (VPT; born at $<30$ weeks of gestation) infants, this critical period of brain development will occur in the ex utero environment, with growing concern that environmental factors in the neonatal intensive care unit
(NICU) may deleteriously have an impact on infant brain development, along with associated implications for neurodevelopmental outcomes (2).

A growing body of literature demonstrates that preterm birth alters the typical trajectory of brain growth and development (3). MRI studies in preterm infants have shown reduced brain volumes associated with intraventricular hemorrhage, bronchopulmonary dysplasia, and postnatal dexamethasone exposure (4-6). Even among preterm infants without clear clinical risk factors, reductions in tissue volumes and brain growth have been demonstrated (7-9), some of which may be related to the rapid brain development occurring ex utero during the period corresponding to the third trimester $(10,11)$. Despite providing valuable insight into brain growth trajectories in preterm infants, the majority of these studies have been cross-sectional MRI investigations at term-equivalent postmenstrual age (PMA).

Although term-equivalent MRI adds to our understanding about the impact of brain injury and altered growth on neurodevelopmental outcomes (12), longitudinal studies assessing brain development across gestational ages are crucial for identifying the onset and progression of structural changes (7). Importantly, longitudinal MRI data can provide insight into critical time points of brain maturation and tissue-specific vulnerability, helping to contextualize trajectories of brain development in relation to clinical and environmental exposures (13). Studies of infants born at varying gestational ages have shown marked linear increases in global and regional brain volumes from 25 to 40 weeks of gestation $(14,15)$. However, few studies have examined these changes using serial observations of the same infants over time, with existing investigations largely limited to two assessments (16-19). Although one group reported growth trajectories using additional serial observations $(20,21)$, these were limited to measures of cortical surface area and total brain growth.

The goal of this exploratory study was to utilize serial structural MRI to examine total and tissue-specific brain growth in a cohort of VPT infants during early development.

\footnotetext{
${ }^{1}$ Department of Pediatric Newborn Medicine, Brigham and Women's Hospital, Harvard Medical School, Boston, Massachusetts; ${ }^{2}$ Department of Pediatrics, Washington University, Saint Louis, Missouri; ${ }^{3}$ Department of Neurology, Boston Children's Hospital, Harvard Medical School, Boston, Massachusetts. Correspondence: Terrie E. Inder (tinder@bwh.harvard.edu) 


\section{Brain growth in the NICU Articles}

Specifically, we aimed to (i) explore brain growth trajectories in VPT infants from birth to term equivalent, and (ii) describe tissue-specific differences in brain growth during the time that coincides with the early and late stages of the third trimester.

\section{METHODS}

\section{Subjects}

Structural MRI scans were collected from two prospective longitudinal cohort studies of VPT infants born at $\leq 30$ weeks of gestational age and admitted to the NICU at St. Louis Children's Hospital between 2007 and 2010. The details of these cohorts have been previously published $(11,22)$. Infants whose data included termequivalent and serial scans were selected for inclusion in this study. Briefly, of the total 137 eligible infants, 99 had term-equivalent scans, of which 81 had two or more scans. Of these, 10 infants were not suitable for analysis because of severe injury (defined as grade 3 or 4 intraventricular hemorrhage or cystic periventricular leukomalacia) (23), one due to severe ventricular dilatation without distinct supratentorial injury, eight due to motion artifact, and a further three due to failed automated segmentation. Of the remaining subjects, 18 VPT infants were available for volumetric analysis at the time of evaluation. Written, informed consent was obtained from parents or guardians before enrollment, and institutional review boards approved all procedures.

\section{Image Acquisition}

All preterm infants were scanned without sedation, and serial MRI scans for each infant were collected approximately every 4-6 weeks based upon clinical status $(<30,30-32,33-36$, and $>36$ weeks of PMA). Before scanning, infants were fed, wrapped securely in warm blankets, outfitted with ear protection, and secured in a papoose bag (Contour Fabrications; CFI Medical Solutions, Fenton, MI). Images were collected on a Siemens Magnetom Trio 3-T scanner using a magnetization-prepared rapid gradient echo $T_{1}$-weighted sequence (repetition time/echo time $1,500 / 3 \mathrm{~ms}$, voxel size $1 \times 0.7 \times 1 \mathrm{~mm}^{3}$ ) and turbo spin echo $T_{2}$-weighted sequence (repetition time/echo time $8,600 / 160 \mathrm{~ms}$; voxel size $1 \mathrm{~mm}^{3}$; echo train length 17 ). Images were interpreted by certified pediatric neuroradiologists (Joshua

Table 1. Participant characteristics

\begin{tabular}{|c|c|}
\hline & VPT $(n=18)$ \\
\hline Gestation at birth, mean (SD), weeks & $26.6(1.4)$ \\
\hline Birth weight, mean (SD), g & $966(214)$ \\
\hline SGA, $n(\%)$ & $2(11)$ \\
\hline PMA at term-equivalent $M R I^{a}$, mean (SD), weeks & $37.9(1.5)$ \\
\hline Weight at term-equivalent scan, mean (SD), g & $2,562(288)$ \\
\hline Female, $n(\%)$ & $7(39)$ \\
\hline Antenatal steroids, $n$ (\%) & $14(78)$ \\
\hline Oxygen at 36 weeks, $n$ (\%) & $6(39)$ \\
\hline Postnatal dexamethasone, $n$ (\%) & $1(6)$ \\
\hline Treated PDA ${ }^{\mathrm{b}}, n(\%)$ & $5(28)$ \\
\hline Necrotizing enterocolitis, $n$ (\%) & $2(11)$ \\
\hline Culture-positive sepsis, $n$ (\%) & $4(22)$ \\
\hline Brain injuryc, $n(\%)$ & $2(11)$ \\
\hline $\begin{array}{l}\text { MRI, magnetic resonance imaging; PDA, patent ductus arteriosus; } \\
\text { menstrual age; SGA, small for gestational age; VPT, very preterm. } \\
\mathrm{a}_{n=15 \text { infants with term-equivalent tissue volumes. }} \\
\text { b Medical or surgical. } \\
\text { 'One infant with Grade II intraventricular hemorrhage, one infant v } \\
\text { noncystic periventricular leukomalacia (23). }\end{array}$ & us; PMA, post- \\
\hline
\end{tabular}

Shimony and Robert McKinstry) and a neonatologist (T.E.I.).

\section{Image Analysis}

A previously established segmentation protocol $(19,24,25)$ comprising sequential image-processing algorithms classified brain tissue into cerebrospinal fluid (CSF), cortical gray matter (GM), and unmyelinated white matter (WM). Briefly, these algorithms are designed to reduce image noise and allow classification of tissue according to joint signal intensities in aligned $T_{1}$ - and $T_{2}$-weighted images and anatomic localization using representative voxels selected as training points (4). Using this technique, anatomic structures with similar image-acquisition characteristics can be correctly classified. Segmentations were subsequently manually edited to classify deep nuclear GM (DNGM; i.e., basal ganglia/thalamus), myelinated WM, and cerebellum. The sum of all tissues (unmyelinated and myelinated WM, cortical GM and DNGM, and cerebellum) was computed, generating the total tissue volume. The sum of all tissues plus CSF was further computed, generating the total intracranial volume (ICV).

\section{Statistical Analysis}

Statistical analysis was performed using STATA 13.0 (StataCorp, TX). Absolute and relative brain tissue growth rates occurring between the earliest MRI scan available and the scan at 33-34 weeks of gestation $(\Delta 1)$, and between the scan at 33-34 weeks of gestation and the term-equivalent MRI scan $(\Delta 2)$ were compared using paired $t$-tests. These time points were chosen as they represent approximately the first and second half of the third trimester. Absolute tissue growth rates $\left(\mathrm{cm}^{3} /\right.$ week $)$ for the periods corresponding to $\Delta 1$ and $\Delta 2$ were defined as brain tissue growth divided by the number of weeks elapsed between scans. Relative tissue growth rates (\%/week) were similarly calculated, but using tissue volumes that were first normalized by the total brain tissue volume (excluding CSF), to allow identification of specific tissue types undergoing rapid or slow growth. Absolute tissue growth for $\Delta 1$ and $\Delta 2$ was further computed as a percentage of ICV growth over the corresponding period, to determine the percentage of the intracranial cavity occupied by each tissue, thereby accounting for variations in head size.

\section{RESULTS}

A total of 51 MRI scans from a total of 18 VPT subjects (gestation range 23-29 weeks, mean 26.6 \pm 1.4 weeks) were available for analysis. Participant characteristics for these subjects are summarized in Table 1. Individual patient-level growth trajectories for total tissue, unmyelinated and myelinated WM, cortical GM, cerebellum, and DNGM were constructed from analysis of these 51 MRI scans (Figure 1).

There was a range in the frequency of scans for the individual cases, with three infants being scanned four times, nine scanned three times, and six scanned twice. Sufficient serial data at the appropriate PMA were available for nine infants before and after the 33-34-week cutoff $(\Delta 1$ and $\Delta 2)$ to allow the a priori-defined calculation of tissue growth. Changes in absolute and relative tissue growth were observed between the periods corresponding to the first (i.e., up to 3334 weeks) and second (i.e., from 33 to 34 weeks to term equivalent) half of the third trimester (Table 2). Specifically, increases were observed in absolute growth over the latter period for total tissue volume ((mean difference $(95 \%$ confidence interval, CI) $4.98 \mathrm{~cm}^{3} /$ week (2.51, 7.45), $P=0.002)$, cortical GM $\left(0.07 \mathrm{~cm}^{3} /\right.$ week $(-0.04,0.17)$, $P=0.0002)$, cerebellum $\left(0.35 \mathrm{~cm}^{3} /\right.$ week $(0.13,0.57)$, $P=0.006)$, and total ICV $\left(3.71 \mathrm{~cm}^{3} /\right.$ week $(0.55,6.86)$, 

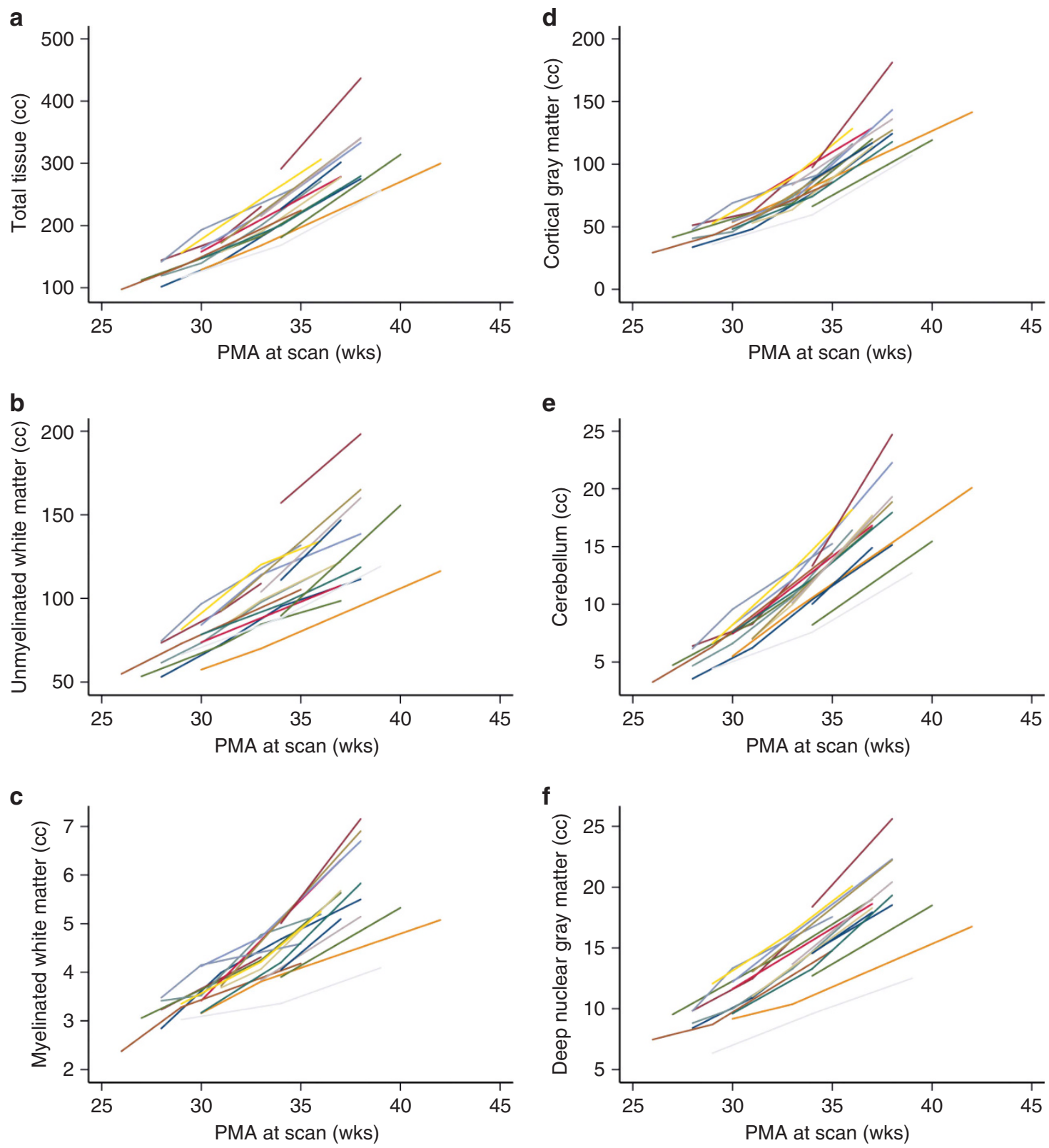

Figure 1. Longitudinal growth trajectories for total tissue, unmyelinated white matter, myelinated white matter, cortical gray matter, cerebellum, and deep nuclear gray matter in preterm infants from 26 weeks of gestation to term-equivalent age. cc, cubic centimeters; PMA, postmenstrual age; wk, week.

$P=0.027)$. WM, CSF, and DNGM maintained consistent absolute growth rates over both periods.

The increased growth rate observed for cortical GM remained significant even after accounting for variations in head size, with the percentage of ICV occupied by cortical GM increasing from $29.4 \%$ in the period before $33-34$ weeks to $47.2 \%$ in the period after $33-34$ weeks $(95 \%$ CI $(10.4,25.3)$, $P=0.001$; Table 3$)$. Unmyelinated WM occupied $20.6 \%$ of ICV after 33-34 weeks vs. an initial 30.6\% (95\% CI $(-19.00$, $-1.05), P=0.033)$. The percentage of ICV occupied by cerebellum increased slightly from 5.5 to $6.1 \%$, although this did not reach significance $(95 \%$ CI $(-0.12,1.21), P=0.093)$. The percentage occupied by myelinated WM and DNGM remained largely unchanged over this period (mean difference (95\% CI), myelinated WM 0.11\% ( $-0.27,0.49), P=0.516$, and DNGM $-1.61 \%(-5.68,2.46), P=0.389))$. The percentage of ICV occupied by CSF decreased by $16 \%$ over this period, but did not reach statistical significance $(95 \%$ CI $(-37.87,5.13)$, $P=0.117)$.

Normalizing individual tissue volumes by total tissue volume (Table 2) revealed differential patterns of relative tissue growth between the periods corresponding to the first and second half of the third trimester. Notably, an accelerated increase in relative cortical GM growth rate was observed after 33-34 weeks (1.6\%/week) compared with an earlier increase of $0.5 \% /$ week $(95 \%$ CI $(0.35,1.85), P=0.010)$. In 


\section{Brain growth in the NICU Articles}

Table 2. Brain tissue growth in VPT infants before and after 33-34 weeks of gestation

\begin{tabular}{|c|c|c|c|c|}
\hline Tissue type & Delta & $\begin{array}{c}\text { Mean } \\
(\mathrm{SD} ; n=9)\end{array}$ & $\begin{array}{l}\text { Mean difference } \\
(95 \% \mathrm{Cl})\end{array}$ & $P$ value \\
\hline \multicolumn{5}{|c|}{ Absolute tissue growth $\left(\mathrm{cm}^{3} /\right.$ week) } \\
\hline \multirow[t]{2}{*}{ Total tissue } & 1 & $15.59(3.63)$ & $4.98(2.51,7.45)$ & 0.002 \\
\hline & 2 & $20.56(2.25)$ & & \\
\hline \multirow[t]{2}{*}{ CSF } & 1 & $8.60(8.17)$ & $-3.05(-9.08,2.97)$ & 0.276 \\
\hline & 2 & $5.55(2.87)$ & & \\
\hline \multirow[t]{2}{*}{ Unmyelinated WM } & 1 & $6.69(2.26)$ & $\begin{array}{c}-1.60 \\
(-3.55,0.36)\end{array}$ & 0.096 \\
\hline & 2 & $5.10(0.91)$ & & \\
\hline \multirow[t]{2}{*}{ Myelinated WM } & 1 & $0.21(0.07)$ & $\begin{array}{c}0.07 \\
(-0.04,0.17)\end{array}$ & 0.174 \\
\hline & 2 & $0.28(0.12)$ & & \\
\hline \multirow[t]{2}{*}{ Cortical GM } & 1 & $6.27(1.43)$ & $\begin{array}{c}5.61 \\
(3.56,7.66)\end{array}$ & 0.0002 \\
\hline & 2 & $11.88(2.26)$ & & \\
\hline \multirow[t]{2}{*}{ DNGM } & 1 & $1.38(1.51)$ & $\begin{array}{c}-0.28 \\
(-1.40,0.85)\end{array}$ & 0.584 \\
\hline & 2 & $1.10(0.31)$ & & \\
\hline \multirow[t]{2}{*}{ Cerebellum } & 1 & $1.20(0.27)$ & $\begin{array}{c}0.35 \\
(0.13,0.57)\end{array}$ & 0.006 \\
\hline & 2 & $1.55(0.37)$ & & \\
\hline \multirow[t]{2}{*}{ ICV } & 1 & $21.75(4.00)$ & $\begin{array}{c}3.71 \\
(0.55,6.86)\end{array}$ & 0.027 \\
\hline & 2 & $25.46(5.40)$ & & \\
\hline \multicolumn{5}{|c|}{ Relative tissue growth (\%/week) } \\
\hline \multirow[t]{2}{*}{ Unmyelinated WM } & 1 & $-0.62(0.48)$ & $\begin{array}{c}-1.02 \\
(-1.77,-0.27)\end{array}$ & 0.014 \\
\hline & 2 & $-1.64(0.59)$ & & \\
\hline \multirow[t]{2}{*}{ Myelinated WM } & 1 & $-0.08(0.03)$ & $\begin{array}{c}0.03 \\
(-0.01,0.07)\end{array}$ & 0.105 \\
\hline & 2 & $-0.04(0.05)$ & & \\
\hline \multirow[t]{2}{*}{ Cortical GM } & 1 & $0.52(0.49)$ & $\begin{array}{c}1.10 \\
(0.35,1.85)\end{array}$ & 0.010 \\
\hline & 2 & $1.62(0.58)$ & & \\
\hline \multirow[t]{2}{*}{ DNGM } & 1 & $0.18(0.87)$ & $\begin{array}{c}-0.28 \\
(-0.96,0.40)\end{array}$ & 0.377 \\
\hline & 2 & $-0.10(0.08)$ & & \\
\hline \multirow[t]{2}{*}{ Cerebellum } & 1 & $0.28(0.09)$ & $\begin{array}{c}-0.11 \\
(-0.19,-0.03)\end{array}$ & 0.013 \\
\hline & 2 & $0.16(0.08)$ & & \\
\hline
\end{tabular}

Delta 1: $\leq 33-34$ weeks; delta 2: $\geq 33-34$ weeks.

$\mathrm{Cl}$, confidence interval; CSF, cerebrospinal fluid; DNGM, deep nuclear gray matter; GM, gray matter; ICV, intracranial volume; VPT, very preterm; WM, white matter.

contrast, the unmyelinated WM growth rate demonstrated a greater relative decrease after $33-34$ weeks, from $-0.6 \%$ to $-1.6 \% /$ week $(95 \%$ CI $(-1.77,-0.27), P=0.014)$. Cerebellar growth showed a reduction in relative increase after 3334 weeks, from $0.3 \%$ to $0.2 \%$ /week ( $95 \%$ CI $(-0.19,-0.03)$, $P=0.013)$ ).
Table 3. Brain tissue growth in VPT infants before and after 33-34 weeks of gestation as a percentage of ICV

\begin{tabular}{lcccc}
\hline Tissue type & Delta & $\begin{array}{c}\text { Mean } \\
(S D ; n=9)\end{array}$ & $\begin{array}{c}\text { Mean difference } \\
(95 \% \mathrm{Cl})\end{array}$ & $\begin{array}{c}P \\
\text { value }\end{array}$ \\
\hline Tissue growth (\% ICV) & & & & \\
Total tissue & 1 & $70.84(13.62)$ & $8.50(-2.14,19.13)$ & 0.103 \\
CSF & 2 & $79.33(7.43)$ & & \\
& 1 & $37.04(28.56)$ & $-16.37(-37.87,5.13)$ & 0.117 \\
Unmyelinated WM & 2 & $20.67(7.43)$ & & \\
& 1 & $30.62(8.03)$ & $-10.02(-19.00,-1.05)$ & 0.033 \\
Myelinated WM & 2 & $20.60(4.92)$ & & \\
Cortical GM & 1 & $0.99(0.32)$ & $0.11(-0.27,0.49)$ & 0.516 \\
& 2 & $1.10(0.40)$ & & \\
DNGM & 1 & $29.42(7.31)$ & $17.82(10.39,25.25)$ & 0.001 \\
& 2 & $47.24(7.48)$ & & \\
Cerebellum & 1 & $5.92(5.23)$ & $-1.61(-5.68,2.46)$ & 0.389 \\
& 2 & $4.31(0.77)$ & & \\
& 1 & $5.53(1.11)$ & $0.55(-0.12,1.21)$ & 0.093 \\
& 2 & $6.08(0.70)$ & &
\end{tabular}

Delta 1: $\leq 33-34$ weeks; Delta 2: $\geq 33-34$ weeks. Cl, confidence interval; CSF, cerebrospinal fluid; DNGM, deep nuclear gray matter; GM, gray matter; ICV, intracranial volume; VPT, very preterm; WM, white matter.

\section{DISCUSSION}

We report for the first time tissue-specific differences in $e x$ utero brain growth trajectories during the period corresponding to the first and second half of the third trimester. Specifically, we observed a marked increase in cortical GM, occupying nearly half of the intracranial cavity after 3334 weeks of gestation, with a greater than threefold increase in relative tissue growth rate over this period. Notably, this was mirrored by an almost threefold decrease in relative unmyelinated WM growth rate, and a $10 \%$ decrease in the proportion of ICV occupied by unmyelinated WM beyond 33-34 weeks. Cerebellar growth rates also differed over this period, with a slightly higher relative growth rate observed before 33-34 weeks of gestation. Together, these findings demonstrate that cortical GM undergoes rapid growth during the latter half of the third trimester, whereas unmyelinated WM and cerebellum undergo faster relative growth during the first half of the third trimester.

The contrasting relative cortical GM and WM trajectories observed here are consistent with cross-sectional observations in preterm infants documenting brain tissue growth between 27 and 45 weeks $(26,27)$. Importantly, our results provide novel evidence for differential ex utero brain growth development taking place in the NICU environment, specifically, the rapid expansion of cortical GM from 33 to 34 weeks to term equivalent. We postulate that the latter half of the third trimester represents a potentially critical period of tissue-specific developmental vulnerability. This hypothesis is corroborated by recent reports of rapid fetal cortical GM growth in the third trimester compared with WM (28), as well as evidence of marked increases in fetal cortical GM growth 


\section{Articles | Mathews et al.}

along with decreases in fetal WM and cerebellar growth during the third trimester (28-39 weeks) compared with the second trimester (18-27 weeks) (1). In their study, Andescavage et al. demonstrated fetal WM as the primary initial contributor to cerebral development peaking between 29 and 30 weeks of gestation, at which point relative cortical GM volume begins to rapidly increase (1). For infants born VPT, this period of accelerated growth taking place ex utero is susceptible to disruption by exposures and stressors in the NICU $(2,29)$, and our previous work suggests that regions of rapid cortical expansion are likely to be more sensitive to postnatal experience and insult (30). Importantly, disruptions to normal cortical maturational trajectories during the late third trimester have been postulated to underpin the neurodevelopmental impairments seen in preterm infants $(20,21,31)$. Related to this, we have reported in the larger cohort the potential adverse effects of varying amounts of environmental sound and language exposure in the NICU, namely reduction in normal hemispheric asymmetry and lower language scores at the age of 2 years in infants from private rooms (11). Although the current study included VPT infants from both private room and open-ward environments, we were unable to assess the associated potential differences in brain developmental trajectories because of our small sample size.

From a biological perspective, our findings are consistent with the dynamically changing processes characteristic of cortical maturation during this period, namely increasing synaptic complexity and dendritic arborization (31). Notably, cortical microstructural development has been shown to be impaired in a dose-dependent manner by preterm ex utero exposure (31), with animal studies suggesting that these alterations may reflect dendritic retraction secondary to perinatal stress (32). More recent animal studies have proposed that neurodevelopmental disturbances in VPT survivors are attributed to cortical growth abnormalities specifically related to reduced dendritic spines and impaired expansion of the dendritic arbor, as well as overall reduced synaptic density $(33,34)$. Relatedly, the greater variability in brain tissue volumes observed at closer to term gestation (Figure 1) may reflect altered growth trajectories secondary to clinical exposures, such as opiates, nutrition, and environmental influences as outlined above.

The relative increase in cerebellar growth observed before 33-34 weeks of gestation is consistent with evidence of the cerebellum's dynamic growth phase during the second and third trimesters (35), characterized by external granule cell proliferation and migration, and formation of the internal granular layer (36). Our observation of a reduction in relative increase in cerebellar growth beyond 33-34 weeks was somewhat unexpected, given previously documented linear increases in cross-sectional investigations of cerebellar volume from 28 weeks of PMA to term (37). Of note, we have previously reported in the larger cohort reductions in transverse cerebellar diameter at term-equivalent age in association with cumulative fentanyl dose (22). Although a large proportion of subjects in the current study received fentanyl (89\%), exposure was very brief, with six out of the nine infants receiving less than 2 days of fentanyl. Nonetheless, given the cerebellum's developmental vulnerability to opioid exposure $(38,39)$, and more recent reports demonstrating associations between increased opioid exposure and impaired cerebellar growth (40), further investigations into these relationships along with longitudinal neurodevelopmental follow-up are needed to establish the clinical significance of these findings.

Despite major advancements in the application of noninvasive MRI to study early brain development in the preterm infant, existing investigations have been largely cross-sectional in nature, or have been employed in limited serial assessments. The primary strength of this study is the use of individual serial, rather than cross-sectional cohort data, allowing us to serially track the progression of brain development early in the neonatal course and identify critical periods of tissue-specific growth. However, due to the difficulties in obtaining multiple serial MRI scans on VPT infants during the period before term equivalent, this approach has resulted in a smaller sample size than some previously reported cross-sectional or longitudinal studies with fewer imaging time points. A further limitation relates to the assumption of linear tissue growth around an apparent inflection point at 33-34 weeks, and larger cohorts with multiple assessments throughout this critical developmental period are needed to enable exponential modeling of brain growth. In addition, because of the sample size of the current cohort, the population was not further stratified based on birth weight or other clinical factors that may be associated with brain growth such as nutrition or duration of ventilation. Although this may be considered as a limitation, it ensures that the data are a true representation of the general population of VPT infants seen in routine clinical practice. Nonetheless, future work in larger cohorts investigating the relationships between differential patterns of growth in brain tissue during the critical NICU period and perinatal risk factors, such as sex, infection, and WM injury, is needed to provide added insight into potential early predictors of deviations in brain development. Furthermore, exploration of brain tissue trajectories in relation to later neurodevelopmental outcomes may identify functional impairments that can be predicted by early deviations from normal brain growth and maturation.

\section{CONCLUSIONS}

Assessment of brain tissue growth trajectories in the NICU has the potential to identify early deviations in brain development before discharge, allowing focused timely interventions such as therapeutic, nutritional, or neurodevelopmental support. This is the first report utilizing serial MRI data to document regional differences in brain tissue growth trajectories ex utero during the period corresponding to the first and second half of the third trimester. Importantly, this study provides novel insight into the maturational vulnerability of the rapidly expanding cortical GM in the NICU, and highlights the importance of continued research into interventions aimed at reducing or ameliorating exposure 


\section{Brain growth in the NICU Articles}

to potentially harmful stressors during this developmentally critical period.

\section{ACKNOWLEDGMENTS}

We gratefully thank Joshua Shimony and Robert McKinstry for image interpretation, as well as the families who participated in the study.

\section{STATEMENT OF FINANCIAL SUPPORT}

This work was supported by the National Institutes of Health (R01 HD057098, R01 HD061619, UL1 TR000448, K23 MH105179, and K02 NS089852), McDonnell Center for Systems Neuroscience, and Intellectual and Developmental Disabilities Research Center at Washington University (P30 HD062171). No honorarium, grant, or other form of payment was given to anyone to produce the manuscript.

Disclosure: The authors declare no conflict of interest.

\section{REFERENCES}

1. Andescavage NN, du Plessis A, McCarter R, et al. Complex trajectories of brain development in the healthy human fetus. Cereb Cortex 2016;27:5274-83.

2. Smith GC, Gutovich J, Smyser C, et al. NICU stress is associated with brain development in preterm infants. Ann Neurol 2011;70:541-9.

3. Ment LR, Kesler S, Vohr B, et al. Longitudinal brain volume changes in preterm and term control subjects during late childhood and adolescence. Pediatrics 2009;123:503-11.

4. Thompson DK, Warfield SK, Carlin JB, et al. Perinatal risk factors altering regional brain structure in the preterm infant. Brain 2007;130:667-77.

5. Murphy BP, Inder TE, Huppi PS, et al. Impaired cerebral cortical gray matter growth after treatment with dexamethasone for neonatal chronic lung disease. Pediatrics 2001;107:217-.

6. Vasileiadis GT, Gelman N, Han VK, et al. Uncomplicated intraventricular hemorrhage is followed by reduced cortical volume at near-term age. Pediatrics 2004;114:e367-72.

7. Bouyssi-Kobar M, du Plessis AJ, McCarter R, et al. Third trimester brain growth in preterm infants compared with in utero healthy fetuses. Pediatrics 2016: 138.

8. Mewes AU, Huppi PS, Als $\mathrm{H}$, et al. Regional brain development in serial magnetic resonance imaging of low-risk preterm infants. Pediatrics 2006;118:23-33.

9. Monson BB, Anderson PJ, Matthews LG, et al. Examination of the pattern of growth of cerebral tissue volumes from hospital discharge to early childhood in very preterm infants. JAMA Pediatr 2016;170:772-9.

10. Lefevre J, Germanaud D, Dubois J, et al. Are developmental trajectories of cortical folding comparable between cross-sectional datasets of fetuses and preterm newborns? Cereb Cortex 2016;26:3023-5.

11. Pineda RG, Neil J, Dierker D, et al. Alterations in brain structure and neurodevelopmental outcome in preterm infants hospitalized in different neonatal intensive care unit environments. J Pediatr 2014;164: $52-60$ e 52.

12. Woodward LJ, Anderson PJ, Austin NC, et al. Neonatal MRI to predict neurodevelopmental outcomes in preterm infants. $\mathrm{N}$ Engl J Med 2006;355:685-94.

13. Lenroot RK, Giedd JN. Brain development in children and adolescents: insights from anatomical magnetic resonance imaging. Neurosci Biobehav Rev 2006;30:718-29.

14. Nishida M, Makris N, Kennedy DN, et al. Detailed semiautomated MRI based morphometry of the neonatal brain: preliminary results. Neuroimage 2006;32:1041-9.

15. Huppi PS, Warfield S, Kikinis R, et al. Quantitative magnetic resonance imaging of brain development in premature and mature newborns. Ann Neurol 1998;43:224-35.

16. Kersbergen KJ, Makropoulos A, Aljabar P, et al. Longitudinal regional brain development and clinical risk factors in extremely preterm infants. J Pediatr 2016;178:93-100 e106.
17. Moeskops P, Benders MJ, Kersbergen KJ, et al. Development of cortical morphology evaluated with longitudinal MR brain images of preterm infants. PLoS ONE 2015;10:e0131552.

18. Young JM, Powell TL, Morgan BR, et al. Deep grey matter growth predicts neurodevelopmental outcomes in very preterm children. Neuroimage 2015;111:360-8.

19. Zacharia A, Zimine S, Lovblad KO, et al. Early assessment of brain maturation by MR imaging segmentation in neonates and premature infants. AJNR Am J Neuroradiol 2006;27:972-7.

20. Kapellou O, Counsell SJ, Kennea N, et al. Abnormal cortical development after premature birth shown by altered allometric scaling of brain growth. PLOS Med 2006;3:e265.

21. Rathbone R, Counsell SJ, Kapellou O, et al. Perinatal cortical growth and childhood neurocognitive abilities. Neurology 2011;77:1510-7.

22. McPherson C, Haslam M, Pineda R, et al. Brain injury and development in preterm infants exposed to fentanyl. Ann Pharmacother 2015;49: 1291-7.

23. Kidokoro H, Anderson PJ, Doyle LW, et al. Brain injury and altered brain growth in preterm infants: predictors and prognosis. Pediatrics 2014;134: e444.

24. Inder TE, Huppi PS, Warfield S, et al. Periventricular white matter injury in the premature infant is followed by reduced cerebral cortical gray matter volume at term. Ann Neurol 1999;46:755-60.

25. Warfield SK, Kaus M, Jolesz FA, et al. Adaptive, template moderated, spatially varying statistical classification. Med Image Anal 2000;4:43-55.

26. Makropoulos A, Aljabar P, Wright R, et al. Regional growth and atlasing of the developing human brain. Neuroimage 2015;125:456-78.

27. Kuklisova-Murgasova M, Aljabar P, Srinivasan L, et al. A dynamic 4D probabilistic atlas of the developing brain. NeuroImage 2011;54:2750-63.

28. Scott JA, Habas PA, Kim K, et al. Growth trajectories of the human fetal brain tissues estimated from 3D reconstructed in utero MRI. Int J Dev Neurosci 2011;29:529-36.

29. Brummelte S, Grunau RE, Chau V, et al. Procedural pain and brain development in premature newborns. Ann Neurol 2012;71:385-96.

30. Hill J, Inder T, Neil J, et al. Similar patterns of cortical expansion during human development and evolution. Proc Natl Acad Sci USA 2010;107: 13135-40.

31. Ball G, Srinivasan L, Aljabar P, et al. Development of cortical microstructure in the preterm human brain. Proc Natl Acad Sci USA 2013;110:9541-6.

32. Murmu MS, Salomon S, Biala Y, et al. Changes of spine density and dendritic complexity in the prefrontal cortex in offspring of mothers exposed to stress during pregnancy. Eur J Neurosci 2006;24:1477-87.

33. Back SA. Cerebral white and gray matter injury in newborns: new insights into pathophysiology and management. Clin Perinatol 2014;41:1-24.

34. Dean JM, McClendon E, Hansen K, et al. Prenatal cerebral ischemia disrupts MRI-defined cortical microstructure through disturbances in neuronal arborization. Sci Transl Med 2013;5:168ra7 5:10.1126/scitranslmed.3004669.

35. Tam EW. Potential mechanisms of cerebellar hypoplasia in prematurity. Neuroradiology 2013;55 (Suppl 2): 41-6.

36. Messerschmidt A, Brugger PC, Boltshauser E, et al. Disruption of cerebellar development: potential complication of extreme prematurity. AJNR Am J Neuroradiol 2005;26:1659-67.

37. Limperopoulos C, Soul JS, Gauvreau K, et al. Late gestation cerebellar growth is rapid and impeded by premature birth. Pediatrics 2005;115: 688-95.

38. Golalipour MJ, Ghafari S. Purkinje cells loss in off spring due to maternal morphine sulfate exposure: a morphometric study. Anat Cell Biol 2012;45:121-7.

39. Hauser KF, Gurwell JA, Turbek CS. Morphine inhibits Purkinje cell survival and dendritic differentiation in organotypic cultures of the mouse cerebellum. Exp Neurol 1994;130:95-105.

40. Zwicker JG, Miller SP, Grunau RE, et al. Smaller cerebellar growth and poorer neurodevelopmental outcomes in very preterm infants exposed to neonatal morphine. J Pediatr 2016;172:81-7 e82. 